\title{
Metastatic malignancies and the effect on arterial stiffness and blood pressure levels: the possible role of chemotherapy
}

This article was published in the following Dove Press journal:

OncoTargets and Therapy

\author{
Eleni Res' \\ Stella Maria Kyvelou ${ }^{2}$ \\ Charalambos Vlachopoulos ${ }^{2}$ \\ Kyriaki Manousou' \\ Dimitris Tousoulis ${ }^{3}$ \\ Christodoulos Stefanadis ${ }^{2}$ \\ Dimitris Pektasides ${ }^{3}$ \\ 'Third Department of Medical \\ Oncology, Agioi Anargyroi General \\ Oncology Hospital of Kifissia, \\ ${ }^{2}$ Cardiology Department, First \\ Cardiology Clinic, Athens Medical \\ School, Hippokration Hospital, \\ ${ }^{3}$ Second Department of Internal \\ Medicine, School of Medicine, \\ University of Athens, Athens, Greece
}

Correspondence: Stella Maria Kyvelou First Cardiology Clinic, Athens Medical School, Hippokration Hospital, 97B Perikleous Street, Athens 10563, Greece Tel +3069728375I8

Email smkyvel@gmail.com
Background: The aim of the prospective study was to evaluate blood pressure (BP) and the arterial stiffness before and after chemotherapy in three subgroups of patients with metastatic colorectal, renal cell, and gastrointestinal carcinoma and exploit, if possible, the effect of chemotherapy and biological agents in the event of cardiotoxicity.

Methods: A total of 171 patients were included in the study: 60 with kidney cancer, 18 with gastrointestinal stromal tumors (GISTs), and 93 with metastatic colorectal cancer. All patients were subjected to full clinical and laboratory evaluation before and after chemotherapy. Arterial-stiffness indices were assessed before the initiation and after the completion of chemotherapy by means of pulse wave velocity (PWV; Complior) and augmentation index (AIx; SphygmoCor).

Results: Patients in all three cancer cohorts exhibited significantly $(P<0.001)$ higher levels of carotid-radial PWV, carotid-femoral PWV, and AIx postchemotherapy, which remained significant after adjustment for BP and body-mass index. AIx exhibited greater change in the bowel-cancer cohort compared to the kidney and GIST cohorts (median 3.6, 1.75, and 1.4, respectively; $P<0.001$ ), which remained significant after adjustment for BP and body-mass index. Multiple regression analysis showed that patients with higher baseline systolic BP, diastolic BP, ejection fraction, and carotid-femoral PWV exhibited smaller differences postchemotherapy, while AIx75 baseline levels showed no difference postchemotherapy.

Conclusion: There is a clear burden in arterial stiffness in patients under chemotherapy for kidney, GIST, and metastatic colorectal cancer, irrespective of BP and other confounders.

Keywords: malignancy, pulse wave velocity, target therapies

\section{Introduction}

Cancer and cardiovascular disease (CVD) are two of the leading causes of worldwide mortality. ${ }^{1}$ Cancer patients may develop CVD secondarily to chemotherapy cardiotoxicity. Recent efforts in cardio-oncology have begun to revise the focus toward identifying CVD early in patients suffering from malignancies, as well as the potential cardiotoxicity of chemotherapy. ${ }^{2,3}$ According to the multiple-hit hypothesis, patients with cancer are exposed to a number of events that together make them more prone to reduced CV reserves, development of CVD, and thus death. ${ }^{4}$ Heart disease following chemotherapy may be the result of either direct CV damage caused by the regime itself or induced atherosclerosis due to cancer treatment-related $\mathrm{CV}$ risk factors. ${ }^{5}$

Colorectal cancer is the second-most common type of malignancy in Europe, whereas $15 \%-25 \%$ of newly diagnosed patients have metastatic disease at diagnosis. 
The disease can be recurrent or lead to distant metastases in up to $50 \%$ of all cases. ${ }^{6,7}$ Survival for patients with metastatic colorectal cancer has overall increased rapidly in the last 20 years, largely due to advances in systemic chemotherapy approaches with newer regimes and the introduction of biological agents. ${ }^{8,9}$ However, the adverse effects of these biological agents, especially in the CV system, have not been fully exploited.

Sunitinib and sorafenib are tyrosine-kinase inhibitors (TKIs) of growth factor receptors, the most important of which are VEGF, PDGF, and Kit, and these are widely used for the treatment of metastatic renal cell carcinoma. ${ }^{10,11}$ Even though these biological agents are implicated in cardiac toxicity, the nature of myocardial damage from TKI treatment has not yet been extensively investigated, with controversial results from studies regarding the possible mechanisms involved and the type of provoked cardiotoxicity.

Imatinib is extremely effective in patients with advanced gastrointestinal stromal tumors (GISTs) through inhibition of the Kit kinase; however, previous data demonstrated that imatinib can lead to significant cardiac dysfunction when administered to mice at clinically relevant dosages. ${ }^{12}$ Studies have reported low incidence of clinically important heart failure in patients with GISTs treated with imatinib. ${ }^{13,14}$ A clear association between imatinib and cardiac toxicity is yet to be confirmed. The aim of the present prospective study was to evaluate blood pressure (BP), ejection fraction (EF), and arterial stiffness before and after chemotherapy in three subgroups of patients with metastatic colorectal, renal cell, and gastrointestinal carcinoma and exploit if possible the effect of chemotherapy and biological agents in the event of cardiotoxicity.

\section{Methods}

The study comprised 171 patients who were divided in to three groups based on the underlying malignancy: 60 with kidney cancer, 18 with GISTs, and 93 with metastatic colorectal cancer. The recruitment of the patients started in 2010 and finished in 2015. The study protocol was revised and approved in 2010 by the Ethics Committee of Hippokration Hospital, Athens, Greece, where the study was conducted. All patients in the study provided written informed consent prior to enrollment.

Full clinical and laboratory evaluation was carried out to exclude patients with recent ( $<6$ months) cerebrovascular events, coronary artery disease, ischemic or nonischemic heart failure, ventricular arrhythmia, sinus bradycardia ( $<55$ beats/minute), sinus tachycardia ( $>100$ beats/minute), and atrioventricular conduction disturbance. Blood samples were collected from the antecubital vein between 8 and $10 \mathrm{am}$, with the patient in the sitting position for at least 10 minutes and at least after an overnight fast. All tests were conducted in the same laboratories using the criteria of the World Health Organization.

\section{Cardiovascular evaluation}

Prior to initiation of chemotherapy and immediately after its completion, all patients were subjected to full CV evaluation.

\section{Blood pressure evaluation}

The diagnosis of arterial hypertension was made based on either systolic BP (SBP; >140 mmHg) or diastolic BP (DBP; >90 $\mathrm{mmHg}$ ) levels on three visits 1 week apart, and mean values were calculated. A 2-week washout period preceded measurement for every patient already receiving antihypertensive treatment. In each visit, BP was measured three times with the patient resting comfortably with back supported in the sitting position after a relaxation period of 10-15 minutes. A mercury sphygmomanometer was used for all measurements with an appropriately sized cuff each time. Twenty-four-hour ambulatory BP monitoring (Spacelabs healthcare, Snoqualmie, WA, USA) was carried out whenever considered appropriate.

\section{Measurement of arterial properties}

Hemodynamic measurements were conducted in the morning in a special room with stable temperature. Subjects were requested to abstain from caffeine, smoking, and alcohol for at least 12 hours before arterial property assessment was performed. SBP, DBP, and heart rate were measured three times in both arms with an automatic mercury sphygmomanometer (M4-I; Omron, Kyoto, Japan).

We used a validated commercially available system (SphygmoCor; AtCor Medical, Sydney, Australia), which uses the principle of applanation tonometry and appropriate acquisition and analysis software for noninvasive recording and analysis of the arterial pulse. High-fidelity micromanometry was used on the nondominant hand's radial artery and gentle pressure applied. After acquiring 20 waveforms, the software performed the analysis, and augmentation index (AIx) values and ascending aortic pressure were obtained.

AI values of the central waveform were measured as indices of wave reflection. AI was defined as augmented pressure divided by pulse pressure and expressed as a percentage. Aortic pulse-pressure velocity was derived by calculating the time between the foot of the pressure wave and the 
inflection point. Higher AI levels suggest increased wave reflection peripherally and/or earlier return of the reflected wave, due to increased pulse wave velocity (PWV; because of increased arterial stiffness) and vice versa. Considering the potential effect of heart rate on AI, all values were automatically corrected for heart rate with SphygmoCor software (AI corrected for heart rate [AIx75]). ${ }^{15,16}$

PWV is the velocity at which the arterial pulse propagates through the circulation, and is, by definition, the distance traveled $(\Delta x)$ by the wave divided by time $(\Delta t)$ that allows the wave to travel that distance. Speed is determined from geometric and elastic properties of the arterial wall. For the calculation of carotid-femoral PWV (PWVc-f), pulse-transit time was measured, as well as the distance traveled between two recording sites $(\mathrm{PWV}=$ distance $[\mathrm{m}] /$ transit time [seconds]) with the Complior device (DuPont, Wilmington, De, USA). Two PWs were measured simultaneously at two sites (at the base of the neck for the common carotid and over the rightfemoral artery) with two transducers. The distance was defined as distance from suprasternal notch to femoral artery minus distance from carotid artery to suprasternal notch. This is generally considered a simple noninvasive technique with good reproducibility. The European Society of Hypertension states that $\mathrm{PWV}>10 \mathrm{~m} / \mathrm{s}$ can be considered an independent marker of end-organ damage, however there is still no universal agreement on fixed PWV-threshold values. ${ }^{17,18}$

\section{Other measurements}

All patients had a baseline electrocardiogram and a repeat one after the completion of chemotherapy. Baseline echocardiographic studies were performed prior to and after the end of chemotherapy cycles. Standard M-mode, two-dimensional, and Doppler images were obtained during breath-hold at end expiration and measurements obtained from the mean of three consecutive beats. Left ventricular systolic and diastolic functions were assessed pre- and postchemotherapy. Echocardiographic studies were performed in the cardiology department by the same operator each time. Tumor markers specific for each malignancy were also assessed before and after the last cycle of chemotherapy.

\section{Chemotherapy}

Sixty patients with kidney cancer were included in the study, 49 of whom received treatment with sunitinib $50 \mathrm{mg}$ for six cycles and eleven with pazopanib $800 \mathrm{mg}$ for six cycles. Eighteen patients with GISTs received treatment with imatinib $400 \mathrm{mg}$ for six cycles. Finally, 93 patients with metastatic colorectal cancer received treatment
Table I Treatment regimen by cancer type

\begin{tabular}{lllll}
\hline $\begin{array}{l}\text { Cancer } \\
\text { type }\end{array}$ & Drug & $\mathbf{n}$ & $\begin{array}{l}\text { Number of cycles } \\
\text { median (range) }\end{array}$ & $\begin{array}{l}\text { Dose (mg) } \\
\text { median (range) }\end{array}$ \\
\hline Kidney & Sunitinib & 49 & $6(3-6)$ & $50(25-50)$ \\
& Pazopanib & 11 & $6(2-6)$ & $800(400-800)$ \\
GIST & Imatinib & 18 & $6(6-6)$ & $400(400-400)$ \\
Bowel & Panitumumab & 93 & $8(3-8)$ & $530(250-900)$ \\
& Oxaliplatin & 44 & $8(4-8)$ & $190(140-480)$ \\
& Irinotecan & 28 & $8(6-8)$ & $343(240-410)$ \\
& Capecitabine & 21 & $6(3-8)$ & $1,300(200-2,000)$ \\
\hline
\end{tabular}

Abbreviation: GIST, gastrointestinal stromal tumor.

with panitumumab-oxaliplatin-based chemotherapy $(n=44)$, panitumumab-irinotecan-based chemotherapy $(n=28)$, and panitumumab-capecitabine-based chemotherapy $(n=21)$ for six to eight cycles. A detailed description of the treatment regimen in each group is presented in Table 1.

\section{Statistical analysis}

Mean (SD), median, and range are used for presenting continuous variables, while categorical variables are presented as frequencies. To evaluate changes in parameters of interest after chemotherapy compared to baseline, the Wilcoxon signed-rank test was used, while for examining differences in these parameters among the three cancer groups, the Kruskal-Wallis test was used. Differences in study parameters among the three cancer types, adjusting for sex, body-mass index (BMI), SBP, and DBP, were assessed using an analysis of covariance model. Finally, for parameters of interest, multiple linear regression analysis was performed. For each of the parameters, the difference (after - before chemotherapy) was used as the dependent variable, while cancer type, sex, BMI, and parameter baseline values were used as independent variables. Parameters examined were SBP (mmHg), DBP (mmHg), AIx75 (\%), PWVc-f (m/second), and carotid-radial PWV (PWVc-r; m/ second). Additionally, within the bowel cancer subgroup, separate analyses were also performed based on the treatment regimen administered: oxaliplatin-based, irinotecan-based, and capecitabine-based. All statistical analyses were performed using $\mathrm{R}$ statistical software. Statistical significance was set at $P=0.05$ (two-sided).

\section{Results}

The study cohort comprised 171 patients divided into three groups according to the underlying malignancy: 60 with kidney cancer, 18 with GISTs, and 93 with metastatic colorectal cancer. Table 1 presents the baseline characteristics of the patients. Hemodynamic measurements and basic 
Table 2 Patient baseline characteristics

\begin{tabular}{|c|c|c|c|c|}
\hline Characteristics & $\begin{array}{l}\text { Entire cohort } \\
(n=|7|)\end{array}$ & $\begin{array}{l}\text { Kidney } \\
(n=60)\end{array}$ & $\begin{array}{l}\text { GIST } \\
(n=18)\end{array}$ & $\begin{array}{l}\text { Bowel } \\
(n=93)\end{array}$ \\
\hline \multicolumn{5}{|l|}{ Age (years) } \\
\hline Mean (SD) & $62.9(10.7)$ & $57.9(10.0)$ & $67.4(6.7)$ & $65.2(10.7)$ \\
\hline Median & 65 & 55.5 & 68.5 & 67 \\
\hline Range & $29-80$ & $38-75$ & $54-79$ & $29-80$ \\
\hline \multicolumn{5}{|l|}{ Sex } \\
\hline Male & 124 (72.5\%) & 42 (70\%) & II (6I.I\%) & $71(76.3 \%)$ \\
\hline Female & $47(27.5 \%)$ & $18(30 \%)$ & $7(38.9 \%)$ & $22(23.7 \%)$ \\
\hline \multicolumn{5}{|l|}{ Surgery } \\
\hline Inoperable & $102(59.6 \%)$ & $13(21.7 \%)$ & $18(100 \%)$ & 89 (95.7\%) \\
\hline Operable & $69(40.4 \%)$ & 47 (78.3\%) & - & $4(4.3 \%)$ \\
\hline \multicolumn{5}{|l|}{ Waist:hip ratio } \\
\hline Mean (SD) & $0.88(0.06)$ & $0.89(0.05)$ & $0.86(0.06)$ & $0.88(0.07)$ \\
\hline Median & 0.9 & 0.9 & 0.88 & 0.9 \\
\hline Range & $0.7-0.95$ & $0.76-0.95$ & $0.70-0.94$ & $0.70-0.95$ \\
\hline \multicolumn{5}{|l|}{ Body-mass index } \\
\hline Mean (SD) & $27.3(6.5)$ & $26.8(6.0)$ & $25.2(7.7)$ & $28(6.5)$ \\
\hline Median & 27.8 & 26.4 & 24.9 & 28.5 \\
\hline Range & $14.3-45.7$ & $15.4-42.5$ & I5.5-45.7 & | $4.3-42.5$ \\
\hline \multicolumn{5}{|c|}{ Cycles of chemotherapy } \\
\hline Mean (SD) & $6.43(1.5)$ & $5.5(1.2)$ & $6.0(-)$ & $7.1(1.5)$ \\
\hline Median & 6 & 6 & 6 & 8 \\
\hline Range & $2-8$ & $2-6$ & $6-6$ & $3-8$ \\
\hline
\end{tabular}

Abbreviation: GIST, gastrointestinal stromal tumor. laboratory results pre- and postchemotherapy are presented in Table 2. Patients with kidney cancer had no significant change in either SBP or DBP levels after chemotherapy, whereas there was a significant change in EF (59.8\% vs $57.8 \%, P=0.001)$, AIx75 (19.03\% vs $20.9 \%, P<0.001)$, PWVc-r (721 m/second vs $7.9 \mathrm{~m} /$ second, $P<0.001)$, and PWVc-f $(7.3 \mathrm{~m} /$ second vs $8.1 \mathrm{~m} /$ second, $P<0.001)$. Patients with GIST had no significant change is SBP, DBP, or EF postchemotherapy; however, AIx75, PWVc-r, and PWVc-f exhibited significant increases postchemotherapy $(17.5 \%$ vs $18.7 \%, P=0.007 ; 7.7 \mathrm{~m} / \mathrm{second}$ vs $8.3 \mathrm{~m} / \mathrm{s}, P<0.001$; $7.4 \mathrm{~m} /$ second vs $8.2 \mathrm{~m} / \mathrm{s}, P<0.001$; respectively). Finally, patients with metastatic colorectal cancer had significant changes in $\operatorname{SBP}(P=0.007)$, EF ( $P=0.007)$, and AIx, PWVc-r, and PWVc-f(17.2\% vs $21.8 \%, P<0.001 ; 7.7$ vs $8.6 \mathrm{~m} / \mathrm{second}$, $P<0.001 ; 7.6 \mathrm{~m} /$ second vs $8.4 \mathrm{~m} /$ second, $P<0.001$; respectively). No significant change was noted in DBP (Table 3).

In Table 4, differences in SBP, DBP, EF, AIx, PWVc-r, and PWVc-f among the three cancer groups are shown. After adjustment for sex, BMI, SBD, and DBP, a significant

Table 3 Selected study parameters before and after chemotherapy by cancer type

\begin{tabular}{|c|c|c|c|c|c|c|}
\hline \multirow[t]{2}{*}{ Parameters } & \multicolumn{2}{|c|}{ Kidney $(n=60)$} & \multicolumn{2}{|c|}{ GIST $(n=\mid 8)$} & \multicolumn{2}{|c|}{ Bowel $(n=93)$} \\
\hline & Before & After & Before & After & Before & After \\
\hline \multicolumn{7}{|l|}{$\mathrm{SBP}(\mathrm{mmHg})$} \\
\hline Mean (SD) & I34.2(I7.4) & I34.6 (I8.9) & I26.5 (I2.7) & I29.8(I5.8) & $137.3(12.3)$ & | 33.5 (| I.7) \\
\hline Median & 135 & 130 & 121.5 & 130 & 140 & 135 \\
\hline Range & $105-169$ & $100-178$ & $110-146$ & $105-150$ & $110-160$ & $105-156$ \\
\hline \multicolumn{7}{|l|}{$\mathrm{DBP}(\mathrm{mmHg})$} \\
\hline Mean (SD) & $72.6(8.9)$ & $70.0(5.8)$ & $72.6(7.6)$ & $74.4(9.7)$ & $73.4(8.3)$ & $72.9(8.2)$ \\
\hline Median & 74.5 & 70 & 70 & 70 & 70 & 70 \\
\hline Range & $60-87$ & $60-82$ & $65-90$ & $65-98$ & $60-90$ & $60-98$ \\
\hline \multicolumn{7}{|l|}{ Pulse } \\
\hline Mean (SD) & $69.5(6.3)$ & $71.6(6.2)$ & $72.3(8.1)$ & $72.8(9.0)$ & $71.6(6.9)$ & $73.3(7.0)$ \\
\hline Median & 70 & 70 & 71 & 70.5 & 70 & 73 \\
\hline Range & $52-86$ & $52-80$ & $55-92$ & $60-96$ & $55-92$ & $60-96$ \\
\hline \multicolumn{7}{|l|}{$\mathrm{EF}(\%)$} \\
\hline Mean (SD) & $59.8(3.4)$ & $57.8(4.3)$ & $58.1(4.6)$ & $57.5(4.9)$ & 59.1 (3.6) & $58.4(4.0)$ \\
\hline Median & 60 & 58 & 60 & 60 & 60 & 60 \\
\hline Range & $55-65$ & $40-65$ & $50-65$ & $45-65$ & $50-65$ & $45-65$ \\
\hline \multicolumn{7}{|l|}{ Alx75 (\%) } \\
\hline Mean (SD) & $19.03(2.42)$ & $20.9(2.8)$ & $17.5(2.2)$ & I8.7 (2.8) & $17.2(2.7)$ & $21.8(4.4)$ \\
\hline Median & 18.95 & 21.3 & 17.3 & 18 & 17.2 & 21.5 \\
\hline Range & | $4.5-25.2$ & I5.6-28.6 & $|4.3-2| .3$ & $14.6-24$ & $12.5-28$ & $14.5-33$ \\
\hline \multicolumn{7}{|l|}{ PWVc-r $(\mathrm{m} / \mathrm{s})$} \\
\hline Mean (SD) & $7.2(0.5)$ & $7.9(0.7)$ & $7.7(0.8)$ & $8.3(1.0)$ & $7.7(0.7)$ & $8.6(1.0)$ \\
\hline Median & 7.2 & 7.9 & 7.7 & 8.1 & 7.6 & 8.5 \\
\hline Range & $6.2-7.9$ & $6.5-9.2$ & $6.5-9.2$ & $6.9-10.6$ & $6.5-10.0$ & $6.9-11.4$ \\
\hline \multicolumn{7}{|l|}{ PWVc-f $(\mathrm{m} / \mathrm{s})$} \\
\hline Mean (SD) & $7.3(0.7)$ & $8.1(0.7)$ & $7.4(0.6)$ & $8.2(0.6)$ & $7.6(0.6)$ & $8.4(0.6)$ \\
\hline Median & 7.2 & 8 & 7.3 & 8 & 7.5 & 8.3 \\
\hline Range & $5.6-8.9$ & $6.5-9.5$ & $6.5-8.6$ & $7.2-9.2$ & $6.3-9.5$ & $7.2-10.7$ \\
\hline
\end{tabular}


Table 3 (Continued)

\begin{tabular}{|c|c|c|c|c|c|c|}
\hline \multirow[t]{2}{*}{ Parameters } & \multicolumn{2}{|c|}{ Kidney $(n=60)$} & \multicolumn{2}{|l|}{ GIST $(n=18)$} & \multicolumn{2}{|l|}{ Bowel $(n=93)$} \\
\hline & Before & After & Before & After & Before & After \\
\hline \multicolumn{7}{|l|}{$\overline{\text { ESR }}$} \\
\hline Mean (SD) & II.8 (10.7) & $12.4(12.0)$ & $7.6(2.1)$ & 9.1 (2.7) & $7.7(2.9)$ & $7.3(2.6)$ \\
\hline Median & 10 & 10 & 7 & 9 & 7 & 7 \\
\hline Range & $5-55$ & 4-95 & $5-12$ & $5-15$ & $5-15$ & $5-15$ \\
\hline \multicolumn{7}{|l|}{ CRP } \\
\hline Mean (SD) & $5.8(3.3)$ & $7.0(4.6)$ & $5.5(1.0)$ & $5.6(0.9)$ & $6.1(7.6)$ & $5.5(2.9)$ \\
\hline Median & 5.3 & 6.1 & 5.3 & 5.3 & 5 & 5 \\
\hline Range & $4-30$ & $4-40.5$ & $4.3-8$ & $4.3-7.5$ & $3.0-77.2$ & $3-30$ \\
\hline \multicolumn{7}{|l|}{ Neutrophils } \\
\hline Mean (SD) & $6.7(4.8)$ & $4.9(3.0)$ & $5.7(2.1)$ & $4.3(2.0)$ & $5.5(3.1)$ & $4.3(2.3)$ \\
\hline Median & 5.3 & 4.3 & 5.4 & 4 & 4.9 & 4 \\
\hline Range & $2.4-28.2$ & $1.2-15.6$ & $1.8-8.9$ & $|.2-8|$. & $1.6-17.3$ & $1.2-12.5$ \\
\hline \multicolumn{7}{|l|}{$\mathrm{Hb}$} \\
\hline Mean (SD) & $12.2(1.3)$ & I I.4 (I.9) & $12.3(1.2)$ & II.0 (I.2) & I2.8 (I.7) & II.6 (I.9) \\
\hline Median & 12.3 & 11 & 12.3 & 10.8 & 12.6 & 11.5 \\
\hline Range & $9.1-14.8$ & $7.5-15.6$ & $10.6-14.6$ & $8.6-13$ & $9.1-17.6$ & $7.9-15$ \\
\hline \multicolumn{7}{|l|}{ Plt } \\
\hline Mean (SD) & $253.3(117.0)$ & $230.1(83.8)$ & $270.3(126.8)$ & $203.9(55.4)$ & $259.4(102.1)$ & $229.9(53.2)$ \\
\hline Median & 240 & 215 & 247.5 & 196.5 & 236 & 219 \\
\hline Range & $116-729$ & $110-446$ & $\mid 45-729$ & $145-350$ & $124-729$ & $130-350$ \\
\hline \multicolumn{7}{|l|}{$\mathrm{CrCl}$} \\
\hline Mean (SD) & 58.4 (II.9) & $58.1(I \mid .7)$ & $63.2(10.9)$ & $63.0(9.9)$ & $64.4(12.4)$ & $60.2(18.0)$ \\
\hline Median & 58 & 56.5 & 59 & 62 & 60 & 60 \\
\hline Range & $30-90$ & $31-86$ & $50.2-89$ & $51.3-86$ & $50.7-112$ & $1.1-112$ \\
\hline \multicolumn{7}{|l|}{$\mathrm{Cr}$} \\
\hline Mean (SD) & $1.3(0.4)$ & $1.3(0.4)$ & I.I (0.2) & $1.2(0.2)$ & $1.0(0.2)$ & $1.2(1.0)$ \\
\hline Median & 1.2 & 1.3 & 1.1 & 1.2 & I & I \\
\hline Range & $0.8-2.6$ & $0.8-2.6$ & $0.8-1.5$ & $0.8-1.5$ & $0.6-1.5$ & $0.6-6$ \\
\hline \multicolumn{7}{|l|}{ Bil } \\
\hline Mean (SD) & $0.7(0.4)$ & $0.8(0.4)$ & $0.6(0.3)$ & $0.7(0.3)$ & $0.6(0.4)$ & $0.8(0.4)$ \\
\hline Median & 0.6 & 0.6 & 0.6 & 0.6 & 0.5 & 0.7 \\
\hline Range & $0.3-1.6$ & $0.3-2.2$ & $0.3-1.6$ & $0.4-1.5$ & $0.1-2.5$ & $0.1-2.2$ \\
\hline \multicolumn{7}{|l|}{ SGOT } \\
\hline Mean (SD) & $24.2(19.8)$ & $34.0(23.4)$ & $20.5(9.8)$ & $21.9(5.3)$ & $24.2(12.8)$ & $22.8(8.1)$ \\
\hline Median & 20 & 25 & 16 & 21.5 & 20 & 21 \\
\hline Range & $10-125$ & $14-120$ & $10-42$ & $15-32$ & $10-86$ & $10-38$ \\
\hline \multicolumn{7}{|l|}{ SGPT } \\
\hline Mean (SD) & $26.0(24.4)$ & $37.3(30.9)$ & $17.6(5.2)$ & $24.8(6.7)$ & $25.4(18.0)$ & $25.9(9.2)$ \\
\hline Median & 20 & 28 & 16 & 24.5 & 17 & 28 \\
\hline Range & $12-166$ & $13-175$ & $|2-3|$ & $15-36$ & $10-84$ & $12-55$ \\
\hline \multicolumn{7}{|l|}{ ALP } \\
\hline Mean (SD) & I08.4 (47.9) & I05.I (42.0) & $79.4(23.4)$ & $82.8(21.4)$ & I56.7 (I55.8) & I 35.6 (95.7) \\
\hline Median & 98 & 93.5 & 78.5 & 77.5 & 111 & 110 \\
\hline Range & $68-352$ & 54-295 & $42-121$ & $52-124$ & $42-926$ & $1 \mid-473$ \\
\hline \multicolumn{7}{|l|}{ CEA } \\
\hline Mean (SD) & I.5 (I.6) & I.5 (I.6) & $1.2(0.4)$ & $1.2(0.3)$ & $98.2(427.0)$ & $59.2(149.1)$ \\
\hline Median & 1.2 & 1 & I & 1 & 7.2 & 5 \\
\hline Range & $0.5-10$ & $0.5-10$ & $0.9-2.3$ & $0.9-2$ & $0.6-4,011.7$ & $0.5-1,097$ \\
\hline
\end{tabular}

Abbreviations: Alx75, augmentation index (heart rate-corrected); ALP, alkaline phosphatase; Bil, bilirubin; CEA, carcinoembryonic antigen; $\mathrm{Cr}$, creatinine; CrCl, $\mathrm{Cr}$ clearance; CRP, c-reactive protein; DBP, diastolic blood pressure; EF, ejection fraction; ESR, erythrocyte-sedimentation rate; GIST, gastrointestinal stromal tumor; Hb, hemoglobin; PIt, platelet; PWV, pulse wave velocity; PWVc-f, carotid-femoral PWV; PWVc-r, carotid-radial PWV; SBP, systolic BP; SGOT, serum glutamic-oxaloacetic; SGPT, serum glutamic pyruvic transaminase.

difference was exhibited among the three cancer types in AIx75 $(P<0.001)$, PWVc-r $(P<0.001)$, and PWVc-f $(P=0.022)$ values at baseline. Comparing the differences (after - before chemotherapy) among the three cancer groups, there were no significant differences in DBP, PWVc-r, or PWVc-f. However, SBP, EF, and AIx exhibited different mean changes in the three cancer subgroups. More specifically, AIx exhibited greater change in the bowel cancer cohort 
Table 4 Differences in SBP, DBP, EF, Alx75, PWVc-r, and PWVc-f before and after chemotherapy in the total cohort and by cancer type

\begin{tabular}{|c|c|c|c|c|c|}
\hline \multirow[t]{2}{*}{ Status } & \multicolumn{3}{|l|}{ Median (range) } & \multirow[t]{2}{*}{$P$-value ${ }^{a}$} & \multirow{2}{*}{$\begin{array}{l}\text { Adjusted } \\
P \text {-value }\end{array}$} \\
\hline & Kidney $(n=60)$ & GIST (n=I8) & Bowel $(n=93)$ & & \\
\hline \multicolumn{6}{|c|}{ Before chemotherapy } \\
\hline SBP & $135(105-169)$ & $121.5(110-146)$ & $140(110-160)$ & 0.013 & $0.012^{\mathrm{b}}$ \\
\hline DBP & $74.5(60-87)$ & $70(65-90)$ & $70(60-90)$ & 0.83 & $0.84^{\mathrm{b}}$ \\
\hline $\mathrm{EF}$ & $60(55-65)$ & $60(50-65)$ & $60(50-65)$ & 0.34 & $0.16^{\mathrm{b}}$ \\
\hline Alx75 & $18.95(14.5-25.2)$ & $17.3(|4.3-2| .3)$ & $17.2(12.5-28)$ & $<0.001$ & $<0.00 \mathrm{I}^{\mathrm{c}}$ \\
\hline PWVc-r & $7.15(6.2-7.9)$ & $7.65(6.5-9.2)$ & $7.6(6.5-10)$ & $<0.001$ & $<0.00 \mathrm{I}^{\mathrm{c}}$ \\
\hline PWVc-f & $7.2(5.6-8.9)$ & $7.25(6.5-8.6)$ & $7.5(6.3-9.5)$ & 0.018 & $0.022^{c}$ \\
\hline \multicolumn{6}{|c|}{ Difference postchemotherapy } \\
\hline SBP & $0(-25$ to 33$)$ & $4.5(-13$ to 33$)$ & $-5(-40$ to I5) & 0.054 & $0.02 I^{b}$ \\
\hline DBP & $-3.5(-24$ to 17$)$ & $2(-10$ to 15$)$ & $0(-25$ to 15$)$ & 0.23 & $0.15^{\mathrm{b}}$ \\
\hline $\mathrm{EF}$ & $0(-20$ to 5$)$ & $0(-5$ to 5$)$ & $0(-5$ to 5$)$ & 0.026 & $0.029^{b}$ \\
\hline Alx75 & $1.75(-1$ to II $)$ & $1.4(-1.7$ to 5.7$)$ & $3.6(0.4-17.2)$ & $<0.001$ & $<0.00 I^{c}$ \\
\hline PWVc-r & $0.6(-0.4$ to 2.2$)$ & $0.4(0.1-1.4)$ & $0.7(0-4.2)$ & 0.09 & $0.085^{c}$ \\
\hline PWVc-f & 0.7 (-0.4 to 2.9$)$ & $0.7(0.1-1.5)$ & $0.8(-0.1$ to 2.4$)$ & 0.93 & $0.81^{c}$ \\
\hline
\end{tabular}

Notes: Values in bold denote significance $(P<0.05)$. Patients with metastatic colorectal cancer were divided into subgroups according to the type of chemotherapy they received: 44 oxaliplatin-based chemotherapy, 28 irinotecan-based chemotherapy, and 21 capecitabine-based chemotherapy. Further analysis of central hemodynamics was performed on the three subgroups, and results are presented in Table 5. Comparison of differences among the three subgroups of chemotherapy showed no significant difference between Alx and PWVc-r; however, PWVc-f exhibited higher mean change in the irinotecan subgroup compared to oxaliplatin and capecitabine in the adjusted model $(0.95[0.2-2.4]$ vs 0.8 [ -0.1 to 1.6$]$ vs 0.5 [0.I-1.7], $P=0.024$, respectively). ${ }^{a}$ Kruskal-Wallis $p$; ${ }^{b} p$-value estimated using ANCOVA with gender and BMI as covariates; cp-value estimated using ANCOVA with gender, BMI, SBP (at baseline) and DPB (at baseline) levels as covariates.

Abbreviations: Alx75, augmentation index (heart rate-corrected); DBP, diastolic blood pressure; EF, ejection fraction; PWV, pulse wave velocity; PWVc-f, carotid-femoral PWV; PWVc-r, carotid-radial PWV; SBP, systolic BP; GIST, gastrointestinal stromal tumor.

compared to the kidney and GIST cohorts (median 3.6, 1.75 and 1.4, respectively; $P<0.001)$. Finally, multiple regression analysis was performed with SBP, DBP, EF, AIx75, PWVc-r, and PWVc-f values before and after chemotherapy (Table 5), which showed that patients with higher baseline SBP, DBP, EF, and PWVc-f values exhibited smaller differences post chemotherapy, while AIx75 baseline levels were not affected postchemotherapy (Table 6).

\section{Discussion}

The main findings of the present prospective study were as follows. Patients with metastatic colorectal cancer, kidney cancer, and GISTs had worse central hemodynamic measurements postchemotherapy. Differences noted in arterial stiffness indices were independent of BMI and BP, whereas patients with metastatic colorectal cancer exhibited a greater increase in AIx compared to GISTs and kidney cancer. In patients with metastatic colorectal cancer, the subgroup of irinotecan-based chemotherapy had higher PWVc-f values postchemotherapy compared to the oxaliplatin and capecitabine subgroups. In all three types of malignancy, patients with higher baseline SBP, DBP, EF, and PWVc-f values exhibited smaller differences postchemotherapy, while AIx75 baseline levels showed no relation to postchemotherapy levels.

To our knowledge, this is the first prospective study to assess arterial stiffness indices pre- and posttreatment with antiangiogenic-based regimens in three malignancy types. Even though there is a positive trend in improved cancerrelated mortality, an emerging increase in CV mortality and morbidity in these patients is also noted. ${ }^{19}$ Though highly effective in the treatment of solid tumors, new anticancer agents that inhibit the VEGF-signaling pathway raise concerns regarding their CV safety. Unfortunately, cancer and CVD share some common pathways; ${ }^{20,21}$ therefore, it is difficult to ascertain whether cancer per se or chemotherapy agents are the predominant causes of $\mathrm{CV}$ complications seen in these patients.

In our study, all arterial stiffness indices increased significantly postchemotherapy in all three types of cancer, whereas AIx 75 showed a greater increase in patients with metastatic bowel cancer compared to patients with kidney and GIST malignancy. The fact that there was a rapid increase in arterial stiffness indices (immediately after six to eight cycles of chemotherapy) suggests a rather acute process (ie, endothelial dysfunction, increase in smooth-muscle tone), rather than a chronic one (ie, atherosclerosis, increased collagen synthesis). This finding is in line with previous studies that investigated mainly the effect of anthracycline chemotherapy on arterial stiffness indices. ${ }^{22,23}$

Sunitinib and pazopanib were used for the treatment of kidney cancer, whereas imatinib was used for GIST patients. These all are TKIs. Panitumumab is an anti-EGFR inhibitor that was used along with standard chemotherapy for patients 
Table 5 Differences in SBP, DBP, EF, Alx75, PWVc-r, and PWVcbefore and after chemotherapy in the subgroup of bowel cancer patients by treatment regimen

\begin{tabular}{|c|c|c|c|}
\hline \multirow[t]{2}{*}{ Variables } & \multicolumn{2}{|l|}{ Median (range) } & \multirow[t]{2}{*}{$P$-value* } \\
\hline & Before & After & \\
\hline \multicolumn{4}{|c|}{ Overall (n=93) } \\
\hline SBP & $140(110-160)$ & $135(105-156)$ & 0.007 \\
\hline DBP & $70(60-90)$ & $70(60-98)$ & 0.63 \\
\hline $\mathrm{EF}$ & $60(50-65)$ & $60(45-65)$ & 0.007 \\
\hline Alx75 & $17.2(12.5-28)$ & $21.5(14.5-33)$ & $<0.001$ \\
\hline PWVc-r & $7.6(6.5-10)$ & $8.5(6.9-11.4)$ & $<0.001$ \\
\hline PWVc-f & $7.5(6.3-9.5)$ & $8.3(7.2-10.7)$ & $<0.001$ \\
\hline \multicolumn{4}{|c|}{ Oxaliplatin-based $(n=44)$} \\
\hline SBP & $140(|10-| 60)$ & $130(105-156)$ & 0.003 \\
\hline DBP & $70(60-90)$ & $70(60-85)$ & 0.71 \\
\hline $\mathrm{EF}$ & $60(55-65)$ & $60(50-65)$ & 0.072 \\
\hline Alx75 & $16.4(12.5-28)$ & $19.5(15.6-32)$ & $<0.001$ \\
\hline PWVc-r & $7.4(6.5-8.6)$ & $8.2(7-11.4)$ & $<0.001$ \\
\hline PWVc-f & $7.5(6.3-8.8)$ & $8.2(7.2-9.1)$ & $<0.001$ \\
\hline \multicolumn{4}{|c|}{ Irinotecan-based $(n=28)$} \\
\hline SBP & $145.5(120-155)$ & $140(\mid 10-156)$ & 0.21 \\
\hline DBP & $80(70-90)$ & $70(60-98)$ & 0.002 \\
\hline $\mathrm{EF}$ & $60(50-65)$ & $60(55-65)$ & 0.57 \\
\hline Alx75 & $18.5(\mid 4.5-23)$ & $23.9(|7.2-3| .5)$ & $<0.001$ \\
\hline PWVc-r & $8(7.4-10)$ & $8.9(8.1-11)$ & $<0.001$ \\
\hline PWVc-f & $7.95(6.5-9.5)$ & $8.6(8.3-10.7)$ & $<0.001$ \\
\hline \multicolumn{4}{|c|}{ Capecitabine-based $(n=2 \mid)$} \\
\hline SBP & $136(120-145)$ & $130(112-150)$ & 0.74 \\
\hline DBP & $69(60-80)$ & $70(60-85)$ & 0.012 \\
\hline $\mathrm{EF}$ & $60(50-65)$ & $60(45-65)$ & 0.057 \\
\hline Alx75 & $17.2(12.5-20)$ & $21.3(14.5-33)$ & $<0.001$ \\
\hline PWVc-r & $7.5(6.9-8.1)$ & $8.5(6.9-9.8)$ & $<0.001$ \\
\hline PWVc-f & $7.2(6.3-8.1)$ & 7.8 (7.5-9) & $<0.001$ \\
\hline
\end{tabular}

Notes: *Wilcoxon signed-rank test. Values in bold denote significance $(P<0.05)$. Abbreviations: Alx75, augmentation index (heart rate-corrected); DBP, diastolic blood pressure; $\mathrm{EF}$, ejection fraction; SBP, systolic blood pressure; PWV, pulse wave velocity; PWVc-f, carotid-femoral PWV; PWVc-r, carotid-radial PWV.

with metastatic colorectal cancer. These new targeted therapies that inhibit the VEGF-signaling pathway (vascular pathway inhibitors) are linked to higher BP levels posttreatment. In our study, a significant increase in SBP was noted only in patients with colorectal metastatic cancer. However, a possible induced arterial stiffening process seen in our study could be explained by the fact that vascular pathway inhibitors could cause a reduction in nitric oxide production, ${ }^{24}$ and increased expression of prohypertensive agents, ${ }^{25}$ such as endothelin 1, also cause microvascular rarefaction, ${ }^{26}$ activate the renin-angiotensin system, ${ }^{27}$ increase oxidative stress, ${ }^{28}$ and induce the pressure-natriuresis system, ${ }^{29}$ leading to alterations in the mechanical properties of large vessels and thus arterial stiffening. Despite the complexity of the mechanisms involved, our data suggest a clear burden of arterial stiffness in these patient's posttreatment, without any other evidence of complementary cardiotoxicity.
Table 6 Multiple regression analysis with SBP, DBP, EF, Alx75, PWVc-f, and PWVc-r pre- and postchemotherapy as dependent variables

\begin{tabular}{|c|c|c|c|}
\hline Variables & $\beta$ & SE $(\beta)$ & $P$-value \\
\hline \multicolumn{4}{|c|}{ SBP difference (after - before) } \\
\hline GIST vs bowel & 3.88 & 2.98 & 0.19 \\
\hline Kidney vs bowel & 3.31 & 1.87 & 0.078 \\
\hline Sex (female) & 3.52 & 1.94 & 0.071 \\
\hline BMI & 0.20 & 0.13 & 0.14 \\
\hline SBP at baseline & -0.30 & 0.06 & $<\mathbf{0 . 0 0 1}$ \\
\hline \multicolumn{4}{|c|}{ SBP difference (after - before) } \\
\hline GIST vs bowel & 1.86 & 1.89 & 0.33 \\
\hline Kidney vs bowel & -2.64 & 1.21 & 0.030 \\
\hline Sex (female) & -0.41 & 1.25 & 0.75 \\
\hline BMI & 0.001 & 0.09 & 0.99 \\
\hline DBP at baseline & -0.70 & 0.07 & $<0.001$ \\
\hline \multicolumn{4}{|c|}{ EF difference (after - before) } \\
\hline GIST vs bowel & -0.14 & 0.80 & 0.87 \\
\hline Kidney vs bowel & -1.19 & 0.51 & 0.021 \\
\hline Sex (female) & -0.96 & 0.53 & 0.071 \\
\hline BMI & -0.07 & 0.04 & $0.07 \mid$ \\
\hline EF at baseline & -0.21 & 0.06 & 0.002 \\
\hline \multicolumn{4}{|c|}{ Alx75 difference (after - before) } \\
\hline GIST vs bowel & -3.28 & 0.72 & $<\mathbf{0 . 0 0 1}$ \\
\hline Kidney vs bowel & -2.66 & 0.49 & $<0.001$ \\
\hline Sex (female) & -0.02 & 0.49 & 0.97 \\
\hline BMI & 0.01 & 0.03 & 0.79 \\
\hline Alx75 at baseline & 0.01 & 0.09 & 0.92 \\
\hline \multicolumn{4}{|c|}{ PWVc-r difference (after - before) } \\
\hline GIST vs bowel & -0.24 & 0.16 & 0.14 \\
\hline Kidney vs bowel & -0.19 & 0.11 & 0.089 \\
\hline Sex (female) & 0.001 & 0.11 & 0.99 \\
\hline BMI & 0.02 & 0.01 & 0.001 \\
\hline PWVc-r at baseline & -0.09 & 0.08 & 0.24 \\
\hline \multicolumn{4}{|c|}{ PWVc-f difference (after - before) } \\
\hline GIST vs bowel & -0.08 & 0.13 & 0.53 \\
\hline Kidney vs bowel & -0.06 & 0.08 & 0.44 \\
\hline Sex (female) & 0.09 & 0.08 & 0.29 \\
\hline $\mathrm{BMI}$ & 0.01 & 0.01 & 0.15 \\
\hline PWVc-f at baseline & -0.40 & 0.06 & $<0.001$ \\
\hline
\end{tabular}

Note: Values in bold denote significance $(P<0.05)$.

Abbreviations: Alx75, augmentation index (heart rate-corrected); BMI, body-mass index; DBP, diastolic blood pressure; GIST, gastrointestinal stromal tumor; PWV, pulse wave velocity; PWVc-f, carotid-femoral PWV; PWVc-r, carotid-radial PWV; SBP, systolic BP; EF, ejection fraction.

In the subgroup of irinotecan-based chemotherapy, along with panitumumab, for metastatic colorectal cancer we noted higher PWVc-f values postchemotherapy compared to capecitabine- and oxaliplatin-based treatments. Irinotecan is a topoisomerase I inhibitor that has been shown to improve survival and quality of life significantly in patients with 5-fluorouracil-resistant disease. ${ }^{30,31}$ Irinotecan is not associated with cardiotoxicity; however, our data suggest a trend toward higher arterial stiffness indices, which could be a point of further investigation in a larger cohort of patients with metastatic colorectal cancer. 
Finally, patients with higher baseline SBP, DBP, and PWV values exhibited smaller differences postchemotherapy, whereas AIx75 was independent of baseline values. Overall, this finding suggests that patients with better baseline central hemodynamics are more prone to an acute effect induced by chemotherapy. Furthermore, AIx is a marker that could potentially be used more objectively for the assessment of arterial stiffening seen in these patients.

The main limitation of the present study is the lack of a control group. However, that would have been unethical and, thus, was not considered an option. The study's strength derives from its blinded characteristics: physicians assessing arterial stiffness were not aware of the type of cancer or patient-treatment regimens, along with the fact that measurements were performed before and immediately after the termination of treatment.

\section{Conclusion}

This prospective study suggests a clear burden in arterial stiffness in patients under chemotherapy for kidney, GISTs, and metastatic colorectal cancer irrespectively of $\mathrm{BP}$ and other confounders. AIx is a better index for such patients, whereas patients with lower baseline BP and arterial stiffness exhibited greater changes. Further, larger studies would need to be conducted to allow generalization of the results.

\section{Acknowledgment}

We would like to acknowledge the contribution of Dr Panagiota Pietri to the conduction of our study.

\section{Disclosure}

The authors report no conflicts of interest in this work.

\section{References}

1. Heidenreich PA, Trogdon JG, Khavjou OA, et al. Forecasting the future of cardiovascular disease in the United States: a policy statement from the American Heart Association. Circulation. 2011;123:933-944.

2. Stone NJ, Robinson J, Lichtenstein AH. 2013 ACC/AHA Guideline on the treatment of blood cholesterol to reduce atherosclerotic cardiovascular risk in adults: a report of the American College of Cardiology/American Heart Association Task Force on Practice Guidelines. Circulation. 2014;129:1-45

3. Lenihan DJ, Cardinale D, Cipolla CM. The compelling need for a cardiology and oncology partnership and the birth of the International CardiOncology Society. Prog Cardiovasc Dis. 2010;53:88-93.

4. Jones LW, Haykowsky MJ, Swartz JJ, Douglas PS, Mackey JR. Early breast cancer therapy and cardiovascular injury. $\mathrm{J} \mathrm{Am} \mathrm{Coll} \mathrm{Cardiol.}$ 2007;50:1435-1441.

5. de Haas EC, Oosting SF, Lefrandt JD, Wolffenbuttel BH, Sleijfer DT, Gietema JA. The metabolic syndrome in cancer survivors. Lancet Oncol. 2010;11(2):193-203.
6. Jemal A, Siegel R, Ward E, Hao Y, Xu J, Thun MJ. Cancer statistics, 2009. CA Cancer J Clin. 2009;2009:225-249.

7. Kindler HL, Shulman KL. Metastatic colorectal cancer. Curr Treat Options Oncol. 2001;2:459-471.

8. Kopetz S, Chang GJ, Overman MJ, et al. Improved survival in metastatic colorectal cancer is associated with adoption of hepatic resection and improved chemotherapy. J Clin Oncol. 2009;27:3677-3683.

9. van Cutsem E, Köhne CH, Hitre E, et al. Cetuximab and chemotherapy as initial treatment for metastatic colorectal cancer. $N$ Engl $J$ Med. 2009;360:1408-1417.

10. Beeram M, Patnaik A, Rowinsky EK. Raf: a strategic target for therapeutic development against cancer. J Clin Oncol. 2005;23:6771-6790.

11. Mendel DB, Laird AD, Xin X, et al. In vivo antitumor activity of SU11248, a novel tyrosine kinase inhibitor targeting vascular endothelial growth factor and platelet-derived growth factor receptors: determination of a pharmacokinetic/pharmacodynamic relationship. Clin Cancer Res. 2003;9:327-337.

12. Kerkelä R, Grazette L, Yacobi R, et al. Cardiotoxicity of the cancer therapeutic agent imatinib mesylate. Nat Med. 2006;12:908-916.

13. Verweij J, Casali PG, Kotasek D, et al. Imatinib does not induce cardiac left ventricular failure in gastrointestinal stromal tumours patients: analysis of EORTC-ISG-AGITG study 62005. Eur J Cancer. 2007;43:974-978.

14. Perik PJ, Rikhof B, de Jong FA, et al. Results of plasma N-terminal pro B-type natriuretic peptide and cardiac troponin monitoring in GIST patients do not support the existence of imatinib-induced cardiotoxicity. Ann Oncol. 2008;19:359-361.

15. Piepoli MF, Hoes AW, Agewall S. Linee guida europee 2016 sulla prevenzione delle malattie cardiovascolari nella pratica clinica [2016 European guidelines on cardiovascular disease prevention in clinical practice]. G Ital Cardiol (Rome). 2017;18(7):547-612. Italian.

16. Boyans V, Ricco JB, Bartelink MEL, et al. 2017 ESC guidelines on the diagnosis and treatment of peripheral arterial diseases, in collaboration with the European Society for Vascular Surgery (ESVS). Rev Esp Cardiol. 2018;71:111.

17. Ioakeimidis N, Tzifos V, Vlachopoulos C, Terentes-Printzios D, Georgakopoulos C, Tousoulis D. Acute effect of coffee on aortic stiffness and wave reflections in healthy individuals: differential effect according to habitual consumption. Int J Food Sci Nutr. 2018;10:1-12.

18. Pietri P, Vlachopoulos C, Terentes-Printzios D, et al. Beneficial effects of low-dose aspirin on aortic stiffness in hypertensive patients. Vasc Med. 2014;19:452-457.

19. Patnaik JL, Byers T, Diguiseppi C, Dabelea D, Denberg TD. Cardiovascular disease competes with breast cancer as the leading cause of death for older females diagnosed with breast cancer: a retrospective cohort study. Breast Cancer Res. 2011;13:R64.

20. Maurea N, Coppola C, Ragone G, et al. Women survive breast cancer but fall victim to heart failure: the shadows and lights of targeted therapy. J Cardiovasc Med (Hagerstown). 2010;11:861-868.

21. Schultz PN, Beck ML, Stava C, Vassilopoulou-Sellin R. Health profiles in 5836 long-term cancer survivors. Int J Cancer. 2003;104: 488-495.

22. Chaosuwannakit N, D' Agostino R Jr, Craig A, et al. Aortic stiffness increases upon receipt of anthracycline chemotherapy. J Clin Oncol. 2010;28:166-172.

23. Drafts BC, Twomley KM, D' Agostino R Jr, et al. Low to moderate dose anthracycline-based chemotherapy is associated with early noninvasive imaging evidence of subclinical cardiovascular disease. JACC Cardiovasc Imaging. 2013;6:877-885.

24. Slamon DJ, Leyland-Jones B, Shak S, et al. Use of chemotherapy plus a monoclonal antibody against Her2 for metastatic breast cancer that overexpresses Her2. N Engl J Med. 2001;344:783-792.

25. Force T, Krause DS, van Etten RA. Molecular mechanisms of cardiotoxicity of tyrosine kinase inhibition. Nat Rev Cancer. 2007; 7: 332-344.

26. Stevenson LW, Perloff JK. The limited reliability of physical signs for estimating hemodynamics in chronic heart failure. $J \mathrm{Am} \mathrm{Med} \mathrm{Assoc.}$ 1989;261:884-888. 
27. Schlessinger J. Common and distinct elements in cellular signaling via EGF and FGF receptors. Science. 2004;306:1506-1507.

28. Holbro T, Hynes NE. ErbB receptors: directing key signaling networks throughout life. Annu Rev Pharmacol Toxicol. 2004;44:195-217.

29. van Cutsem E, Peeters M, Siena S, et al. Open-label phase III trial of panitumumab plus best supportive care compared with best supportive care alone in patients with chemotherapy-refractory metastatic colorectal cancer. J Clin Oncol. 2007;25:1658-1664.
30. Rougier P, van Cutsem E, Bajetta E, et al. Randomised trial of irinotecan versus fluorouracil by continuous infusion after fluorouracil failure in patients with metastatic colorectal cancer. Lancet. 1998;352: $1407-1412$.

31. Cunningham D, Pyrhonen S, James RD, et al. Randomised trial of irinotecan plus supportive care versus supportive care alone after fluorouracil failure for patients with metastatic colorectal cancer. Lancet. 1998;352:1413-1418.

\section{Publish your work in this journal}

OncoTargets and Therapy is an international, peer-reviewed, open access journal focusing on the pathological basis of all cancers, potential targets for therapy and treatment protocols employed to improve the management of cancer patients. The journal also focuses on the impact of management programs and new therapeutic agents and protocols on

\section{Dovepress}

patient perspectives such as quality of life, adherence and satisfaction. The manuscript management system is completely online and includes a very quick and fair peer-review system, which is all easy to use. Visit http://www.dovepress.com/testimonials.php to read real quotes from published authors.

Submit your manuscript here: http://www.dovepress.com/oncotargets-and-therapy-journal 